\title{
Hypermedia information systems in industry
}

\section{by Richard Growder, Wendy Hall, Gary Wills, Dave Humphreys and Jon Ash}

\author{
The requirements for industrial strength hypermedia are well known. \\ If hypermedia applications are to be used successfully in the industrial \\ environment, then considerable effort is required to integrate them \\ into the organisation's current business practices. This implies that \\ any proposed model must be simple to maintain and implement, \\ as well as bringing real benefits to the organisation as a whole. \\ This article discusses the development of such a system, and its \\ implementation and evaluation to support manufacturing operations \\ at Pirelli Cables, Eastleigh.
}

A $\mathrm{s}$ reported in a previous issue of Computing \& Control Engineering Journal, ${ }^{1}$ we developed a hypermedia application for Pirelli Cables, Aberdare, South Wales. The application developed was targeted in particular at maintainers and operators. A main objective of the project was to interconnect all the relevant information about an item, which is spread over a large number of individual documentation. The user could click on an electrical part in a circuit diagram and access all the other documents that contained that part, along with other information, such as location diagrams, parts lists etc. In Fig. 1, the user has selected the object identified as ' $\mathrm{Y} 145$ ' from an electrical drawing and the hypermedia system returns a list of destinations by searching the available link databases.

The Aberdare application considered the information specific to the one machine and was self-contained. In particular it was not possible to link to external resources such as production information and the stores inventory. In order for hypermedia systems to be used widely in industry, it was recognised that the hypermedia application must operate enterprise wide, and not solely concern an isolated piece of equipment. The solution, in part, is the incorporation of additional functionality into the hypermedia system, such as:

- The ability to query remote information systems, for example equipment suppliers, to obtain the latest updates in maintenance procedures.
- A direct interface with the equipment, allowing the hypermedia system to provide the correct supporting information, depending on the equipment's status:

This article considers the problems associated with the development of a very large hypermedia information system suitable for industrial application, and considers the recently completed Factory Information Resource Management (FIRM) project. This was undertaken in order to examine the problems of, and provide solutions to, dealing with capturing, structuring and delivering information on a factory-or organisation-wide basis. The application was developed for Pirelli Cables, using its Supertension sheathing line at Eastleigh, Hampshire, as the target application. A schematic of the $90 \mathrm{~m}$ long line is shown in Fig. 2. The cable moves from right to left, where the crossed-linked polyethylene outer-sheath is extruded onto the cable. In addition to the extruder the line includes a bitumen tank, two caturpullars, cooling tank, spark tester and pay-off and take-up stands. The line can take cable drums up to 50 tonnes containing cables up to $155 \mathrm{~mm}$ diameter, and $40-50 \mathrm{kgm}^{-1}$ in weight. Due to the scope of the application, the hypermedia application not only involved Pirelli, but also Nokia-Maillefer the line builder, and a number of its suppliers, in particular Eurotherm Drives and Siemens.

\section{The FIRM project}

The Aberdare case study highlighted some of the problems with developing an industrial hypermedia 


\section{INFORMATION SYSTEMS}

Fig. 1 Links in the Aberdare application

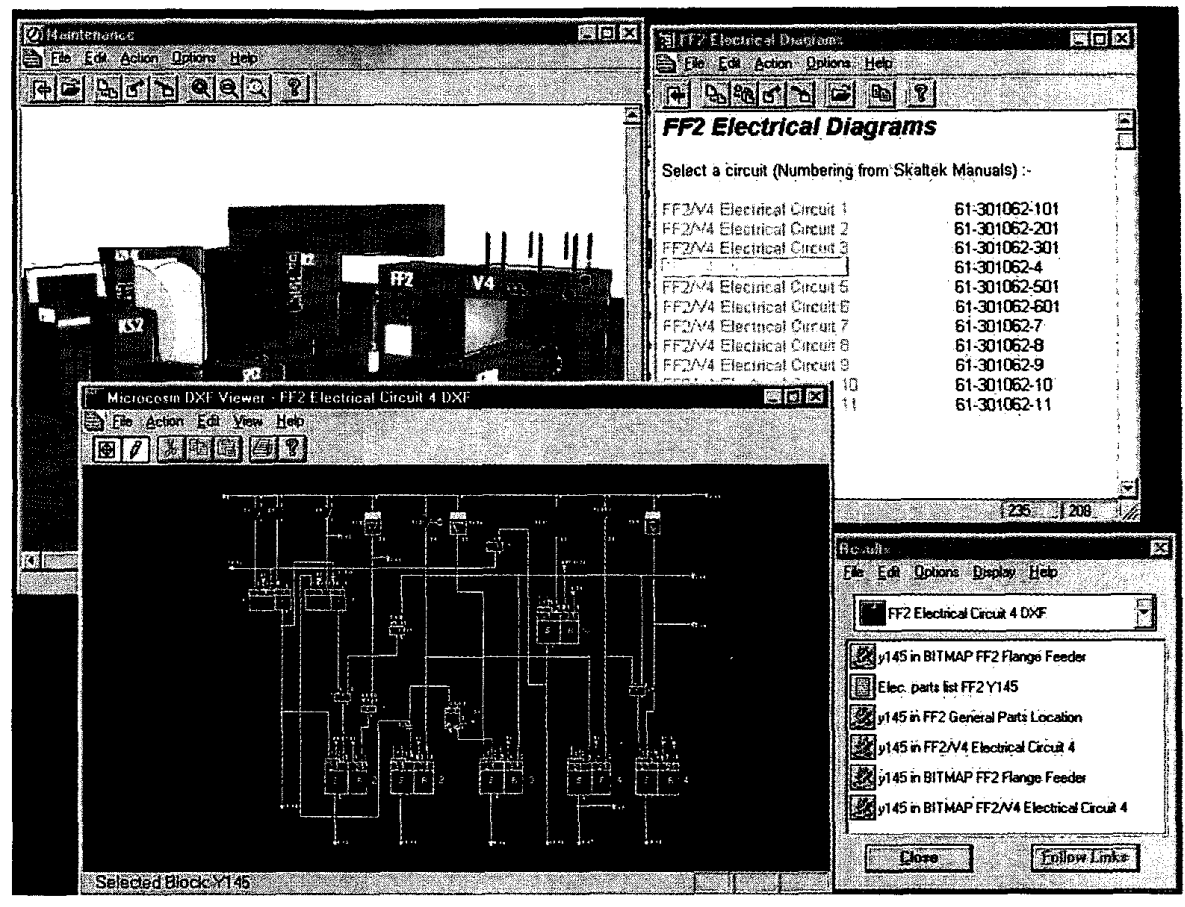

system but only concerned with a single piece of plant. The information was manageable, although it required extensive processing, as it existed only in paper form. In addition the application was also easily controlled, as the hypermedia application was self-contained.

The requirement for industrial strength hypermedia is well known. ${ }^{2}$ If hypermedia is to be used in manufacturing industry, then a considerably amount of work is required to integrate the developed hypermedia system into the existing business processes. Many organisations have methodologies in place to control the flow and quality of information, which need to be embraced by the hypermedia system if it is to be adopted. This means any proposed model must be simple to maintain and implement whilst providing a real benefit for the organisation as a whole.

When using the hypermedia application across the organisation, a large range of users with vastly differing computing skills have to be supported, together with controlling the access to information. In addition the volume of information available will be greater, hence filtering of the information based on the user's job function or specific requirements is essential to stop them being overwhelmed with information.

\section{The FIRM authoring model}

The Aberdare hypermedia resources were approximately 150 Mbyte in size with 7000 links connecting some 600 documents. Although some of the links were automatically generated, the application was starting to become unmanageable, both to expand and manage.
To assist with the development and maintenance of very large industrial hypermedia applications, we have developed a number of tools, some of which will be discussed in this article. As with the Aberdare work, this application was developed using Microcosm, the open hypermedia system developed at the University of Southampton.

\section{Automatic structural linking}

One of the conclusions of the original Aberdare case study was that a great deal of work was required to manually link information. The use of Microcosm's generic links where possible reduced this effort but the building of large-scale hypermedia systems was a large undertaking. Therefore, tools were developed to derive structure semi-automatically or automatically from the information itself. There have been many approaches to this task. The information might be marked up in some structured format, such as SGML (Standard Generalised Mark-up Language) and then links derived from that structure using tools such as Dynatext. However, the application developed in the FIRM system must be capable of supporting the use and structuring of thirdparty information supplied in a variety of formats. Any tools must deal with these formats and so it was decided to develop a simple solution to generate simple, structural links. Most of the links created consisted of navigational shortcuts, providing access to information as follows:

- The table of contents to the relevant section. 


\section{INFORMATION SYSTEMS}

- A list of tables (or figures) to the relevant table (or figure) and their reference in the text.

- Explicit references to other procedures, data sheets etc., be it on the local or the external network.

Microcosm supports the creation of links between documents without modifying the underlying content, and so we were able to readily use the information supplied by companies without conversion. To automatically generate such structural links, tools were developed to process the structure of the documents. As the documents were generated electronically, the structure could be derived from word processor mark-up. Because the documentation supplied was designed for display on the printed page and not the screen, a limited amount of format related conversion work was undertaken to make the resulting electronic version more readable. The developed tool resulted in the document being dissected into small nodes by dividing it into separate sections as defined by the original author. Tables of contents were then automatically generated and linked to these nodes.

Automatic generation of links is made easier by the use of templates/procedures and guidelines for construction of the documents. In practice, several templates/ procedures and guidelines are required, i.e. for manuals, memos, reports, specifications etc. In many companies, these will already exist, either as part of the company's quality procedures or just good practice.
Modular hypermedia applications

In order to make the development of very large-scale hypermedia applications possible a move away from monolithic information sets is required.

A standard principle of software engineering suggests that a large programming problem can be more easily managed by decomposing it into smaller modules that are more easily dealt with. This approach can also be applied to the information domain, so that large information systems are broken down into smaller hypermedia applications, termed modular hypermedia applications (MHAs), each of which represents a fraction of the whole. For example, one MHA could be created describing a motor drive unit used on various production lines whereas another describes the programmable logic controller (PLC).

In Microcosm, an application is defined to be a collection of links documents and processes that, when operated together, can be considered an information system. The MHA model extends this definition to also include a set of child MHAs that provide additional information. Each MHA is considered to be selfcontained (i.e. it does not contain explicit references to documents that are not a part of the MHA). However, an MHA can use generic links to provide context-free linking from anywhere within the resulting application.

If explicit links are required between MHAs then they are defined within the MHA that is the parent of the source and destination. This is because the parent MHA

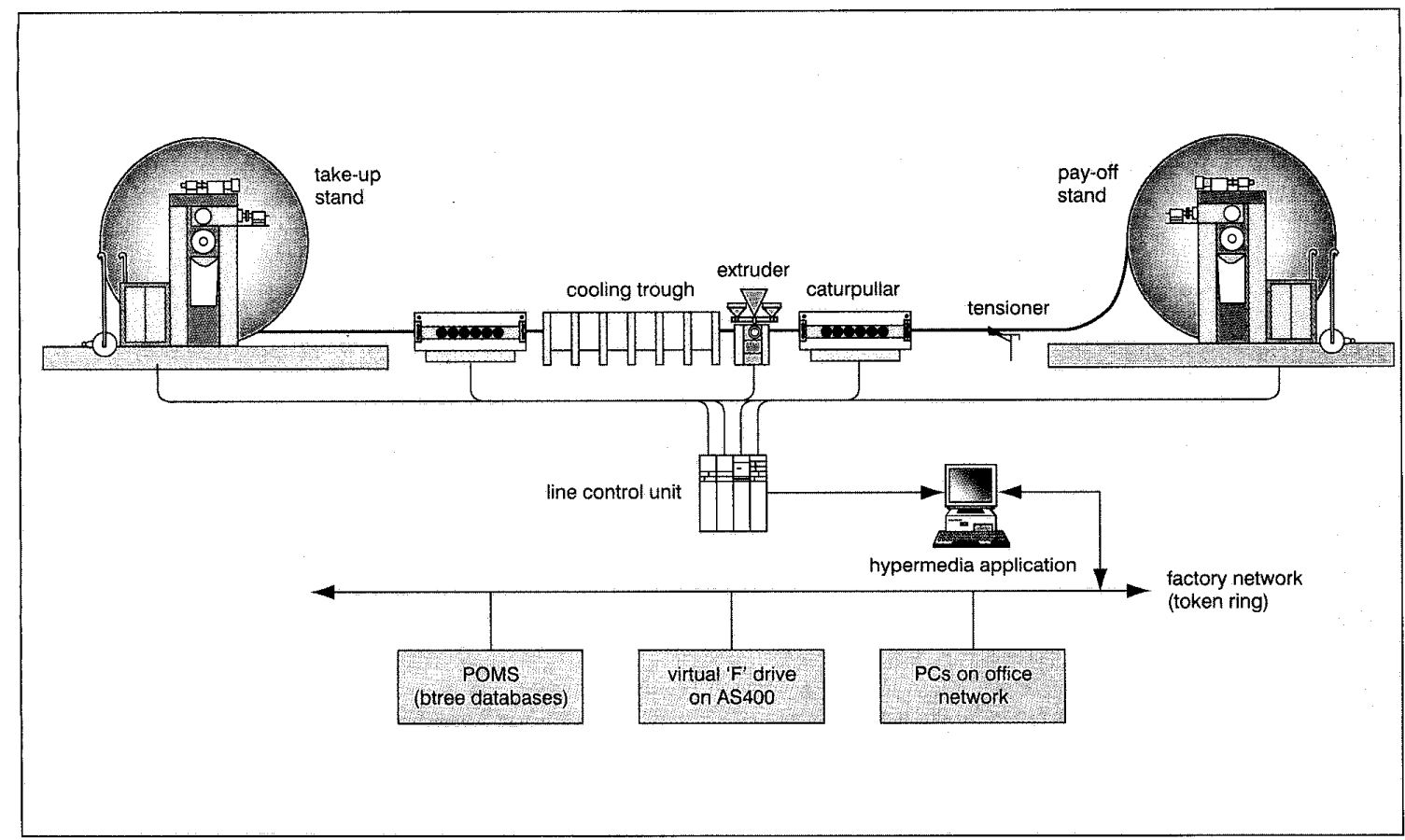

Fig. 2 Eastleigh supertension sheathing line, showing the hypermedia system and its interconnection to external resources 


\section{INFORMATION SYSTEMS

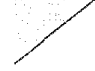

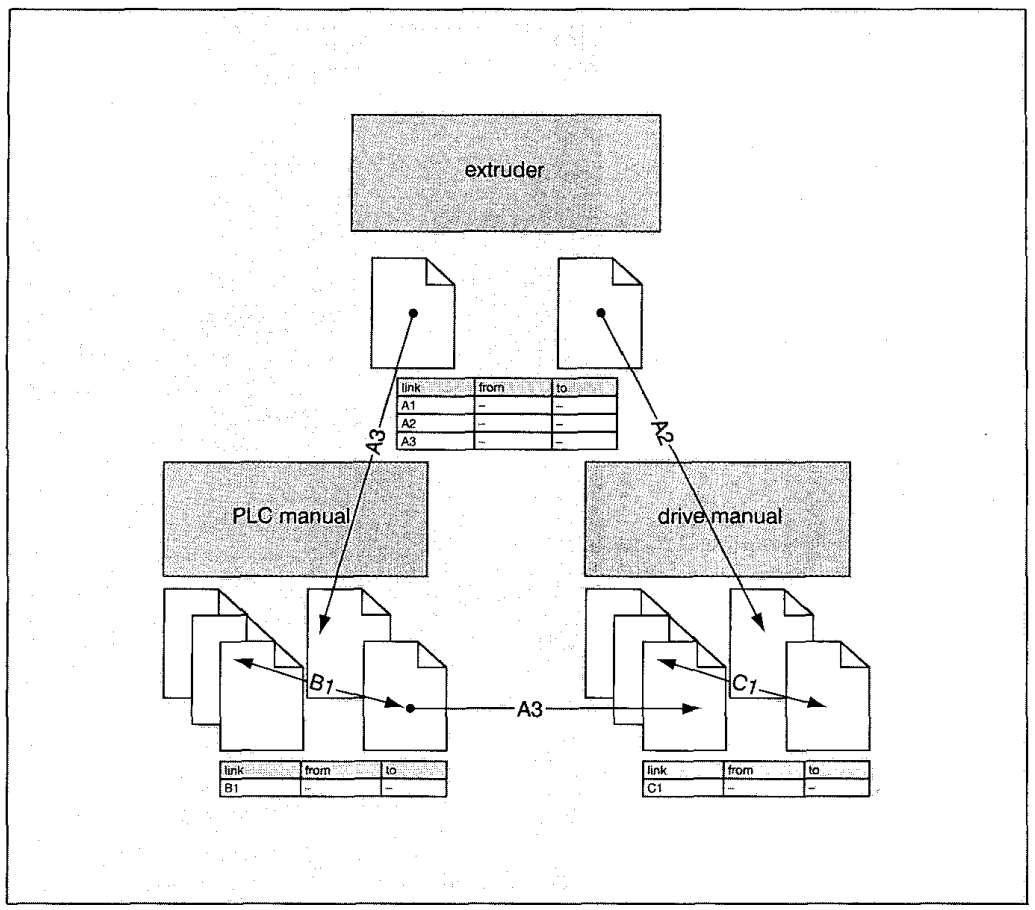

Fig. 3 Example of the MHA relationships

is considered to provide the context in which the two child MHAs are being used. An example of the hierarchical nature of MHAs is shown in Fig. 3. Here the 'extruder' MHA also uses a 'PLC manual' MHA and a 'drive manual' MHA to supplement the available information. As the 'extruder' MHA is providing the context for the use of the other two MHAs, it also holds any cross-MHA links (e.g. link 'A3').

Information reuse becomes critical as the size of hypermedia systems becomes ever larger. The approach described has the benefit in that, once an MHA has been developed, it can then be used in many different contexts. Rather than dealing with reuse at the document level, the author can reuse entire hypermedia collections.

A second benefit is that the modules can be managed centrally, which helps increase the maintainability and quality of the final application. If the content of a module is updated, all uses of that module will also be updated automatically.

Finally, the authoring effort required for building applications becomes less, as they can be built largely from pre-existing information modules. We envisage that, in the future, component manufacturers will be able to supply the documentation in the form of modules that can then be integrated into the corporate information base and used wherever needed.

Link clusters

The original Aberdare case study used Microcosm's generic links to represent higher-level information about an element of the line. This had the advantage that it was easy to implement as it used features already present in the hypermedia system. However, this implementation had several drawbacks. Only one term could be used as a lead-in to the cluster, as the same term was used to provide the interconnectivity. In many cases, several terms were used to describe a component. On a drawing a component may be identified as 'Y145' and on a parts list identified by the manufacturer's part number, ' $62-1221-4598$ ', in the original implementation a copy of the cluster had to be created for each term required. The visibility of clusters was also important. Originally, all clusters were visible, regardless of who was using them, which results in information overload as the application grows. This was because the clusters were represented as links and, as such, the author had no control over their visibility. Finally, each cluster was an information island. It contained a set of documentation relevant to the particular concept it represented, but that was all. In some cases, it would have been useful to be able represent relationships between the various clusters.

All the problems outlined above could be traced back to the original implementation chosen to represent the clusters. As clusters were represented as links and did not exist as first class objects within the information system, it was difficult to provide the additional functionality required to solve these problems. Clearly, the representation and implementation of the clusters would have to be changed.

Electronic thesauri provide an established model for representing clusters of related information and the semantic relationships between them. A thesaurus entry contains one or more lead-ins, which are terms used to locate entries within the thesaurus. The entry has a set of related documents, which are defined to be associated with the entry in question. This representation has the advantage that the terms used to locate a cluster are separate from the documents related to that cluster, which was not the case using the link representation.

There has been much work in the development of better methods for representing the interrelationships between semantically similar documents. However, care must be taken when considering the use of any of these

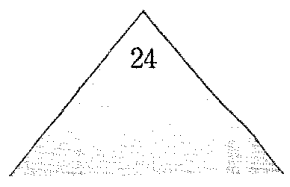


models due to practical considerations of the target application (in this case, manufacturing industry). Rather than building a system explicitly for knowledge representation, the link clusters are simply providing another view of the underlying information.

The simplest method for representing relationships was to define an explicit connection (or link) between the clusters. Each link was a directional relationship that was typed to allow the development of future querying tools. The description generated for traversal of the link in each direction was configurable (for example an information link $A \rightarrow B$ would generate 'more detail on $B$ ' when viewed from $A$, and 'other related information in $\mathrm{A}^{\prime}$ ' when viewed from $B$ ). The author was free to extend the set of available cluster link types.

The final model for link clusters as used in the FIRM application is shown in Fig. 4 and consists of the following:

- A set of source anchors, termed lead-ins, which connect from the information to a particular link cluster (e.g. 'Sel i' as shown in Fig. 4). A lead-in may be generic, i.e. not bound to a particular location within a document. A number of different clusters may use the same lead-in.

- A set of destination documents that are somehow associated with this cluster (determined by the author). Documents may be referenced from several clusters (e.g. both cluster 1 and cluster 3 refer to document A).

- A set of arbitrarily typed relationships between this cluster and other clusters.

A simple permission model has been developed for the clusters to provide a means for controlling their visibility. Users of the system were assigned to groups, which initially reflected the different job descriptions within the company (for example, maintenance, operation, sales etc.). Access control lists were attached to the clusters, allowing the author to control access by specifying an arbitrary list of permissions for users and/or groups. Thus, clusters that represented maintenance information could be tagged to be visible only if the user was a member of the maintenance group.
A simple relational database was used to store the cluster definitions, using tables to represent the relationships, lead-ins and related documents. For the initial implementation, this database was 'compiled' to a Microcosm linkbase, as this required the minimum amount of development work to test the concepts.

Each lead-in became a generic link which, when traversed, caused another linkbase query to be executed to return the relationships and related documents. Cluster relationships were also represented as special links that would cause the destination cluster to be displayed, again by sending a linkbase query. The related documents were 'normal' Microcosm links that caused the relevant information to be displayed. Thus, the user would navigate the link clusters in exactly the same way as they would navigate structural links.

Using this approach, it was possible to experiment easily with the link cluster model. However, there were problems, the biggest being that the links have to recompiled every time the underlying cluster database changes. For the system to be truly usable, the cluster database would have to be queried 'live', and the results presented somehow. Fortunately, the Microcosm system

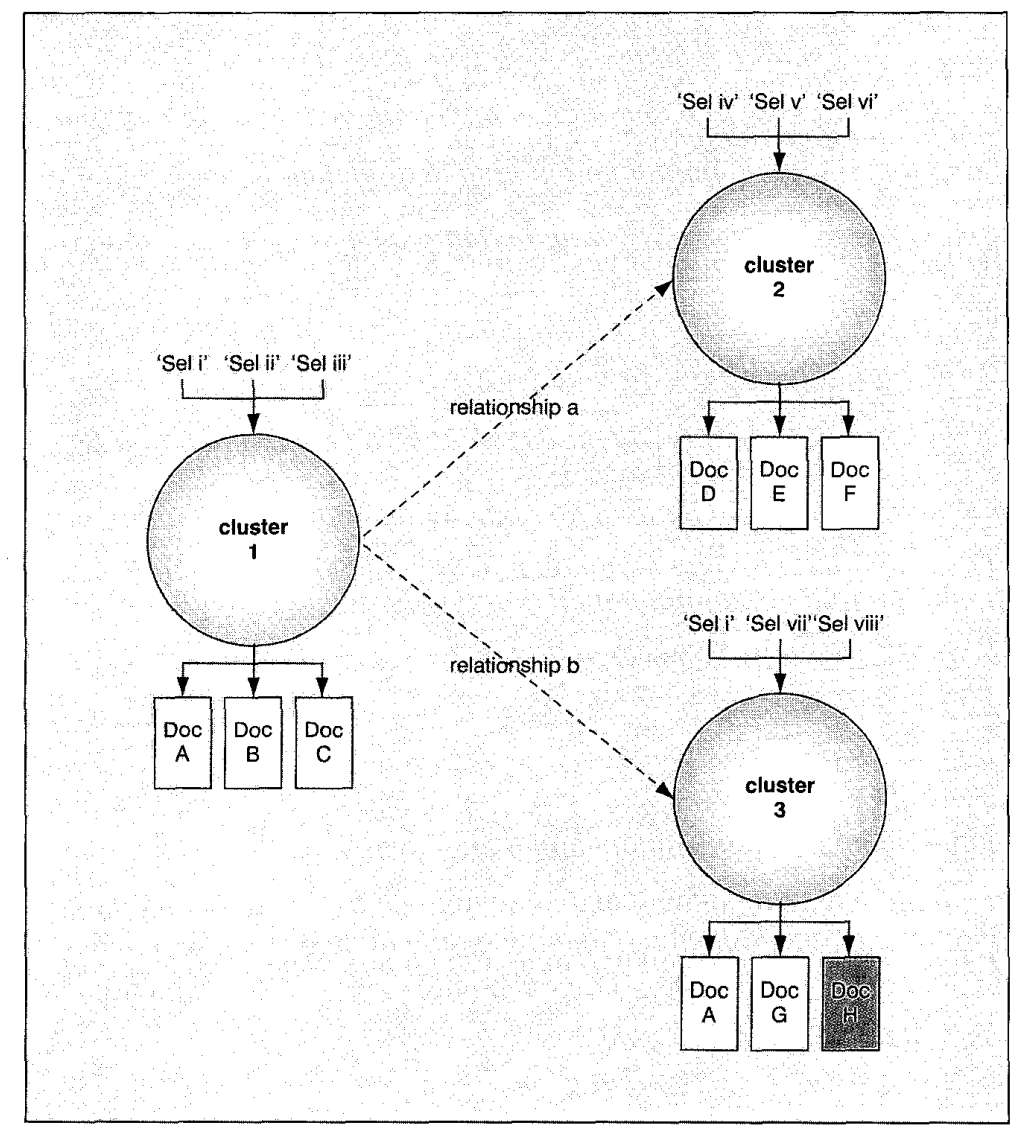

Fig. 4 Example of the link cluster model 


\section{INFORMATION SYSTEMS}

Fig. 5 Page of information.under the control of SHEP

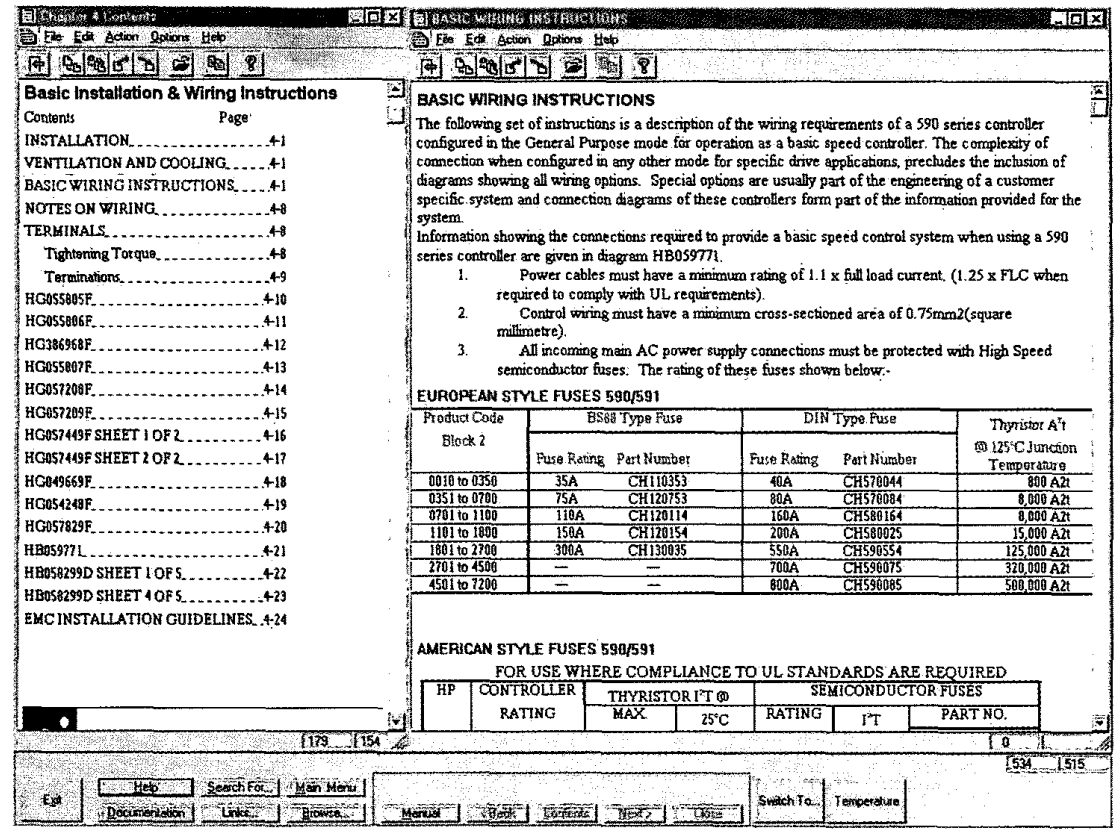

provides an open method for extending the functionality of the information environment. Developers are able to write processes, termed filters, which can extend or modify the system in some way. A cluster database filter was developed for just such a purpose. Again, the results were presented alongside the normal linking interface but this time the cluster links were being dynamically generated. Thus it was possible to modify the underlying database and have those modifications instantly available to the user.

\section{Interfacing with the external resources.}

In order to achieve an organisational information resource, it is essential that the system is able to communicate with external information resources. Each manufacturing area within the Eastleigh plant has a designated virtual drive. The hypermedia application can read the directory structure, file names within the directory, and automatically generate links to any files placed in the directory structure. This allowed the production engineering department to place electronic documents into the system for the operators to read or enter data. Typical documents are the latest version of the line set-up spreadsheet, and additional technical information that would previously have been given on paper. This ensured the operators had the latest set-up and process information and that additional information did not get lost. One of the main management systems used by Pirelli is POMS (Production Operation Management System). All information relating to the process line is stored under the appropriate unique numeric identifier, including drawing lists, materials lists and part information. The hypermedia application interfaces with the databases and presents the user with the information, in a similar format to the existing POMS displays. However, rather than having to $\log$ in and out of different systems, the user can navigate seamlessly between the hypermedia application, the virtual drive and POMS.

As part of the application development an interface was developed to communicate to the line's programmable logic controller; this requires additional software for the PLC and the hypermedia application. The PLC is able to download diagnostic status information, permitting circuit diagrams, faultfinding trees etc. to be pre-loaded, ready for the maintenance technician to carry out fault-finding tasks. In addition various control parameters can be read on a continuous basis, allowing analogue displays to be include in the hypermedia application.

\section{Industrial user interfaces}

The industrial environment brings together users with different and varying computer skills, all of whom need to use the system. Many industrial interfaces try to provide a common look and feel for the user interfaces by providing the same user interface for all applications and user groups. In practice, what is actually required is an appropriate interface for the task, that has a common look and feel yet allows the different group of users, with different abilities, access to the appropriate information.

As the system runs under the Microsoft Windows environment, a number of processes can have interfaces on the screen at the same time. The interfaces all act independently of each other in terms of screen management and can therefore be made to overlap or obscure one another; this may cause problems with inexperienced 


\section{INFORMATION SYSTEMS}

users. Similarly, inexperienced users may have problems with basic screen management, such as inadvertently moving windows off the screen, inability to restore a minimised window etc. Hence, inexperienced users can soon clutter the screen, losing track of where they are going or, worse still, move the document off the viewing area never to be seen again.

A solution for tackling the problems created by having users with differing abilities has been designed and implemented. ${ }^{4}$ An architecture, which promotes the disclosure of state information by the individual processes and allows screen management processes to modify the state of the interface components (the Screen Handler Enabling Process, SHEP), has been developed. SHEP gives the developers of hypermedia applications flexibility when creating interfaces for users of different and varying ability when using Microcosm. The SHEP solution allows the designers of industrial hypermedia applications to provide:

- A rigid framework to support novice users, yet at the same time allow more experienced users to turn off parts of the automatic screen management system, in order to provide a more flexible environment (Fig. 5).

- A system that can be configured to reflect the different user groups. Different tool bars can be used to support the different user categories or different task functions. Similarly, the menus bars associated with the viewers can be designed separately to reflect the different user requirements. These customised menu bars are 'attached' to the viewer by the SHEP system, allowing the developers to provide a common look and feel to the user interface, without the need to provide exactly the same interface functionality.

\section{User evaluation}

Following its development the FIRM resource base comprises over 822 nodes in $26 \mathrm{MHAs}$, resulting in $642 \mathrm{Mbytes}$ of information. Over 5000 links of various types were authored and stored in 90 different linkbases. Compared to the Aberdare application the increase in documents being stored, without the corresponding increase in application size, was largely due the Aberdare's engineering drawing being predominantly bitmap.

All the supertension sheathing line maintainers, operators and managers were trained to use the developed system. Their experience with the application was measured both qualitatively and quantitatively through questionnaires and usability trials. In general comments were very positive; for example, one of the maintainers commented that, in their experience of trying to locate information during a breakdown situation, the hypermedia system was a considerable improvement over existing systems. The questionnaires were used three times, following initial training and after the first and second usability trials. The usability trials were conducted six and twelve months from the installation and initial training of the users. The assessments follow the criteria specified by Neilsen ${ }^{5}$, and are summarised in Table 1 . The responses were normalised between 0 (strongly negative) to 1 (strongly positive), and are shown in Fig. 6. These results show clearly that the users were highly supportive of the introduction of a hypermedia system onto the factory floor.

At the same time users were timed while completing a number of tasks using the current paper-based system, and the hypermedia based system. The tasks were developed to be representative of the operators or

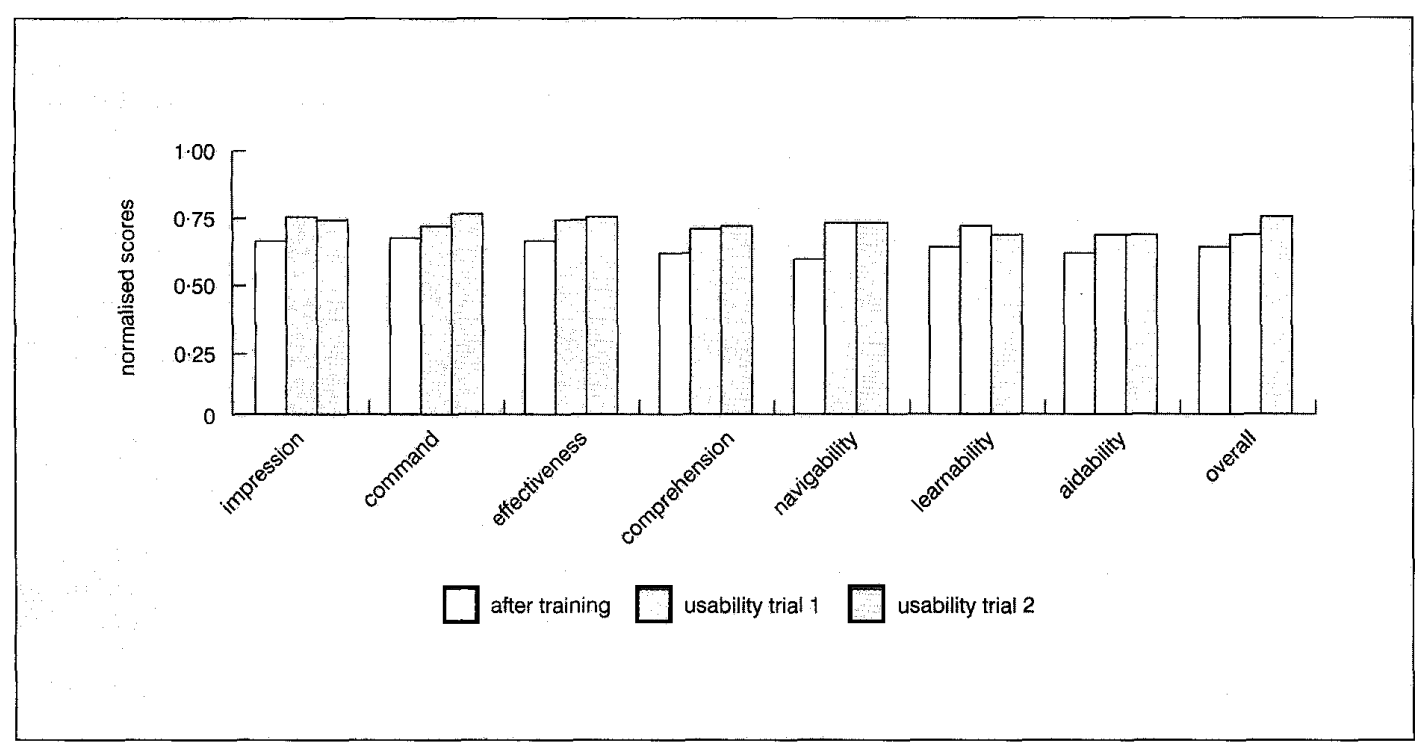

Fig. 6 Results from the questionnaires 


\section{INFORMATION SYSTEMS}

Table 1 Questionnaire categories

\begin{tabular}{|c|c|}
\hline criterion & definition \\
\hline $\begin{array}{l}\text { impression } \\
\text { command } \\
\text { effectiveness } \\
\text { navigability } \\
\text { learnability } \\
\text { aidability } \\
\text { comprehension }\end{array}$ & $\begin{array}{l}\text { the user's feelings about using the } \\
\text { application } \\
\text { the measure to which the user feels that } \\
\text { they are in control } \\
\text { the degree to which the user feels that } \\
\text { they can complete the task using the } \\
\text { software } \\
\text { the degree to which the user can move } \\
\text { around the software } \\
\text { the degree to which the user feels that the } \\
\text { application is easy to become familiar with } \\
\text { the degree to which the application assists } \\
\text { the user to resolve a situation } \\
\text { the degree to which the user understood } \\
\text { the interaction with the application }\end{array}$ \\
\hline
\end{tabular}

Table 2 Time trial results. The time is that taken to complete a predefined task

\begin{tabular}{|c|c|c|c|}
\hline task & media type & mean time, $s$ & ttest \\
\hline first time trial & $\begin{array}{l}\text { paper } \\
\text { hypermedia }\end{array}$ & $\begin{array}{r}1470 \\
51.0\end{array}$ & $p<0.001^{* \ldots}$ \\
\hline second time trial & $\begin{array}{l}\text { paper } \\
\text { hypermedia }\end{array}$ & $\begin{array}{l}137.5 \\
45.3\end{array}$ & $p<0.001^{* * *}$ \\
\hline
\end{tabular}

maintainers normal activities. The results are summarised in Table 2 . The results showed that there was a statistically significant difference in the time to locate information using the current paper-based system and the hypermedia system. In general it was concluded that the users were able to find the information in about one-third of the time when using the hypermedia system. This reduction could be accounted for by the fact that they had to visit fewer documents, and got lost less often using the hypermedia system. A group of apprentices who were unfamiliar with the line were able to locate information with relative ease using the hypermedia system, but became quickly disoriented with the paperbased system.

\section{Concluding remarks}

This article has considered the introduction of an advanced industrial strength hypermedia application at Pirelli Cables at Eastleigh. The work has resulted in the

Table 3 Key benefits

not having to publish and distribute the paper copies of documents

reduce documentation control effort, while ensuring that the users have the correct issue of any document

reduction of errors due to easier cross-referencing between different information resources and easier maintenance of document cross-referencing, after a document is revised

the legibility of the documents is improved

allowing easier system integration, by linking across different information systems

easier access to information from any point in the factory development of tools to help the authoring and delivery of large systems for factory floor use. The evaluation results showed that the introduction of these systems was welcomed by factory floor personnel, and led to significant time saving. While the reduction by twothirds of the time taken to locate specific information, it should be noted that this is dependent on the existing information structure on the factory floor. It would be expected that the difference in time to retrieve information would depend on a company's current practices. We considered that industrial hypermedia systems would give a reduction in time, together with other benefits, some of which are summarised in Table 3.

At present, general interest in the use of advanced hypermedia systems to support in practical industrial situations is cautious, as this technology is seen as 'new', although it has been commercially available for a number of years. However, this attitude is changing and the perceived risk associated with using hypermedia technology is decreasing, especially with the increased use of the World Wide Web, online documentation and electronic commerce.

Therefore, as the need for managing and navigating the information in an industrial environment increases, the demand for industrial hypermedia will increase. Industrial hypermedia, however, is not a panacea for all of industry's information management problems.

\section{Acknowledgments}

The authors acknowledge Pirelli Cables, Eurotherm and the Ford Motor Company for allowing their personnel to take part in the trials, and the EPSRC (Engineering and Physical Science Research Council) for funding the work under grant number GR/L/ 10482

\section{References}

1 CROWDER, R. M., HALL, W, HEATH, I., BERNARD, R, and GASKELL, D. A.: 'Hypermedia maintenance information system', Computing \& Control Engineering Journal, June 1996, 7, (3), pp. $121-128$

2 MALCOLM, K. C., POLTROCK S. E., and SCHULER, D: 'Industrial strength hypermedia: requirements for a large engineering enterprise', Proceedings of Hypertext '91, Seattle, December 1991

3 HSU, L. H., PEIYA L., and DAWIDOWSKY, T. A.: 'Multimedia authoring in the large environment to support complex product documentation', Multimedia Tools and Applications, 1999, 8, pp. 11-64

4 HALL, W., WEAL, M, HEATH, I., WLLS, G. B., and CROWDER, R. M.: 'Flexible interfaces in the industrial environment', International Conference on 'Managing enterprises-stakeholders, engineering, logistics and achievement (ME-SELA9 7)', Loughborough, UK, 22nd24th July 1997, pp.453-460

5 NEILSEN, J.: 'Finding usability problems through heuristic evaluation', CHI '92 Conference, ACM Press, 1992

\section{(C) IEE: 2001}

Richard Crowder, Wendy Hall and Gary Wills are with the Intelligence, Agents and Multimedia Research Group, Department of Electronics and Computer Science, University of Southampton, UK \{rmc, wh, gbw\} @ecs.soton.ac.uk. Dave Humphreys is with Corporate Technical Services, Pirelli Cables, Eastleigh, Hampshire, UK. Jon Ash is with Eurotherm Drives, Littlehampton, UK, jon.ash@drives.eurotherm.co.uk 